${ }^{1}$ Centro Cardiovascular, Hospital Clínico Regional de Concepción: "Dr. Guillermo Grant Benavente". Concepción, Chile. 2Departamento de Cirugía, Facultad de Medicina, Universidad de Concepción. Concepción, Chile. anterno Medicina, Facultad Medicina, Universidad de Concepción. Concepción, Chile.

Los autores no declaran conflictos de interés.

Trabajo no recibió apoyo financiero.

Recibido el 31 de julio de 2017, aceptado el 28 de diciembre de

Correspondencia a: Dr. Roberto González Lagos Departamento de Cirugía, Facultad de Medicina, Universidad de Concepción, Janequeo esquina Chacabuco S/N, Concepción, Chile. rgonzalezlagos@udec.cl

\section{Tratamiento quirúrgico de la úlcera penetrante de aorta ascendente. Casos clínicos}

\author{
ROBERTO GONZÁLEZ L. ${ }^{1,2}$, ALECK STOCKINS L., ${ }^{1,2}$, \\ ENRIQUE SEGUEL S..$^{1,2}$, ANDRÉS JADUE T. ${ }^{1}$, ALEJANDRA RIQUELME U. ${ }^{2}$, \\ JORGE CANALES Z. ${ }^{\mathrm{a}}$, ALBERTO FUENTES E. ${ }^{\mathrm{a}}$, EMILIO ALARCÓN C. ${ }^{1,2}$

\section{Penetrating ulcer of the ascending aorta treated surgically. Report of two cases}

Penetrating aortic ulcer (PAU) is an ulceration of an atherosclerotic plaque which disrupts the aortic internal elastic lamina and that can evolve to an intramural hematoma (IH), aortic dissection or aortic rupture. We report two cases with PAU. A 73 year-old woman with a history of hypertension, presented with acute chest pain of three days of evolution and a hypertensive emergency. An electrocardiogram showed ST-segment elevation and elevated cardiac enzymes. Computed tomography (CT) scans showed an acute ascending aortic mural hematoma secondary to PAU and hemopericardium. Replacement of ascending aorta was performed with a vascular prosthesis with extracorporeal circulation (ECC). PAU and IH were confirmed by histopathology. A 62-year old woman with a history of hypertension, diabetes and chronic obstructive pulmonary disease, consulted for abdominal pain of two days of evolution. A thoracic-abdominal CT scan visualized an uncomplicated PAU and an abdominal aortic aneurysm. Because of abdominal pain and signs of peritoneal irritation, an exploratory laparotomy was performed finding a typhlitis. Resection and ileo-ascendo-anastomosis were performed. A new CT scan showed PAU with high risk of rupture without a clinical acute aortic syndrome. The patient was operated replacing the ascending aorta with a vascular prosthesis using ECC. Three PAU were found. Histopathology confirmed the diagnosis. Both patients evolved without postoperative complications.

(Rev Med Chile 2017; 145: 1490-1494)

Key words: Aorta; Aortic Diseases; Thoracic Surgery.

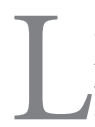
a úlcera penetrante de aorta (UPA) es una patología que forma parte del síndrome aórtico agudo (SAA) junto a la disección aórtica (DAo) y al hematoma intramural (HI $)^{1}$. Se caracteriza por la ulceración de una placa aterosclerótica que afecta a la lámina elástica interna y puede progresar hacia un HI, a una DAo, si existe paso de sangre hacia la capa media o hasta una rotura aórtica ${ }^{2}$.

La incidencia del SAA es de 3 casos por cada 100.000 habitantes al año, de los cuales, $80 \%$ co- rresponden a DAo, $15 \%$ HI y $5 \%$ a UPA ${ }^{3}$. La UPA se localiza principalmente en la porción media y distal de la aorta torácica descendente (62-90\%) o en arco aórtico $(3,4-25 \%)$, mientras que aproximadamente solo $1,8 \%$ corresponde a la aorta ascendente $(\mathrm{AA})^{4}$.

La UPA comúnmente se observa en sujetos de la séptima década de vida que presentan comorbilidades como hipertensión arterial (HTA), diabetes mellitus, enfermedad pulmonar obstructiva crónica (EPOC), insuficiencia cardiaca y renal, 
además, usualmente existe una larga historia de tabaquismo e hiperlipidemia ${ }^{5,6}$. En pacientes más jóvenes, la UPA puede asociarse con enfermedades del tejido conectivo como el síndrome de Marfan o la enfermedad de Ehlers-Danlos ${ }^{7}$.

Se presentan dos casos clínicos de úlcera penetrante de AA (UPAA), un caso de una paciente que debutó como SAA y un segundo caso de una paciente asintomática desde el punto de vista cardiovascular.

\section{Casos clínicos}

\section{Caso 1}

Mujer de 73 años, con antecedentes de HTA, consultó en hospital de origen por dolor retroesternal intenso con valor de 10 en la escala numérica análoga (con valor de 10 en la escala numérica análoga), de tres días de evolución. Se solicitó electrocardiograma (ECG) y enzimas cardíacas, las que resultaron dentro de rangos normales, por lo que se decidió tratamiento ambulatorio con analgésicos. A los dos días, la paciente consultó nuevamente en la urgencia por persistencia del dolor, se realizó ECG que demostró supradesnivel del segmento ST en pared inferior y enzimas cardíacas elevadas. Se diagnosticó infarto agudo de miocardio (IAM), fue evaluada por Cardiología quienes decidieron no trombolizar por el tiempo de evolución. Por persistencia de los síntomas y la emergencia hipertensiva que cursaba, se solicitó radiografía de tórax y una angio-tomografía computada (Angio-TC) la que demostró: HI agudo de la AA secundario a úlcera parietal con signos de sangrado y hemopericardio (Figura 1A, B y C). Debido a los hallazgos, la paciente fue catalogada como SAA y derivada a nuestro centro para su resolución quirúrgica.

La paciente ingresó ese mismo día sin deterioro hemodinámico, la ecocardiografía transesofágica mostró hallazgos similares a los descritos en el Angio-TC, por lo que el equipo de cardiocirugía

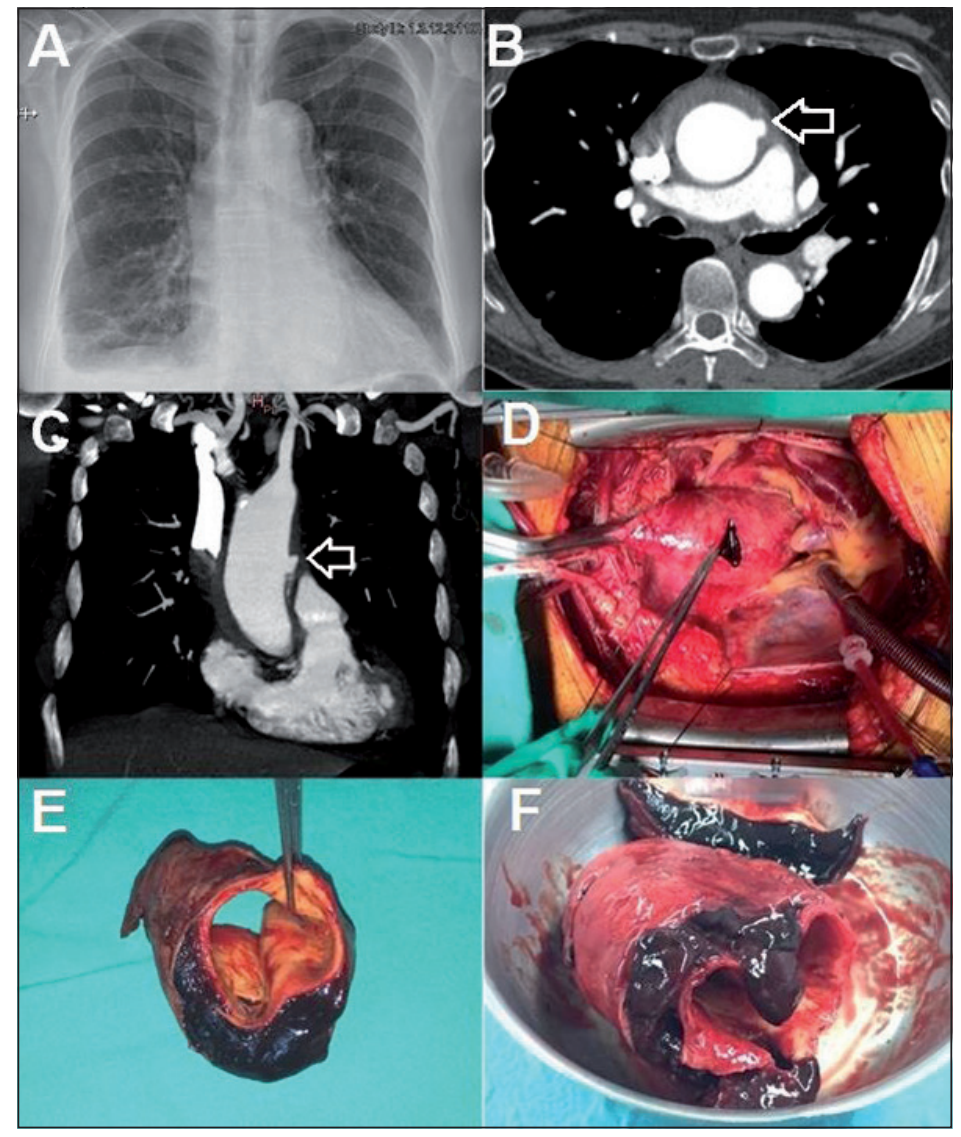

Figura 1. A: Radiografía de tórax de ingreso. B-C: Angio-TC tórax que evidencia úlcera penetrante de aorta ascendente (flecha). D-E-F: Imágenes intraoperatoria que evidencian rotura de úlcera penetrante de aorta ascendente y hematoma intramural. 
decidió cirugía de urgencia. Se abordó por esternotomía y se realizó con circulación extracorpórea (CEC) reemplazo de AA con prótesis de dacron

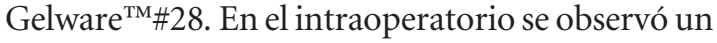
hemopericardio a tensión de aproximadamente $300 \mathrm{cc}$, un hematoma aórtico ascendente que no involucraba el arco aórtico, una úlcera penetrante con rotura contenida, sin observar disección aórtica (Figura 1D, E y F).

La paciente evolucionó favorablemente en el postoperatorio, sin requerimientos de drogas vasoactivas, solo presentó fibrilación auricular, la cual revirtió con el uso de amiodarona. Fue dada de alta a los diez días de postoperada.

El estudio anatomopatológico confirmó UPAA y HI. Actualmente, a un año de seguimiento, la paciente continúa sus controles sin presentar sintomatología cardiovascular.

\section{Caso 2}

Mujer de 62 años, con los antecedentes de HTA, diabetes mellitus tipo 2 y EPOC secundario a tabaquismo, consultó en su hospital de origen por dos días de evolución de dolor abdominal intenso asociado a vómitos. Se realizó un estudio ecográfico y tomografía computada (TC) que visualizó un aneurisma de la aorta abdominal (diámetro de $5,6 \mathrm{~cm}$ ) y una UPAA no complicada, por lo que se derivó a nuestro hospital.

Ingresó a nuestro centro y se descartó que el dolor fuera secundario a una complicación del aneurisma abdominal, se realizó ecografía abdominal que sugirió proceso infeccioso en fosa iliaca derecha. Con los antecedentes reunidos, se catalogó como abdomen agudo apendicular y se realizó una laparotomía exploradora encontrándose tiflitis y necrosis intestinal, por lo que se decidió efectuar una resección e íleo-ascendo-anastomosis.

Posteriormente, debido al tiempo transcurrido, se realizó nuevo Angio-TC que mostró UPAA, con paso de contraste que se extendía más allá de la pared aórtica (Figura $2 \mathrm{~A}, \mathrm{~B}$ y C), sin clínica de SAA. Se efectuó estudio preoperatorio con coro-

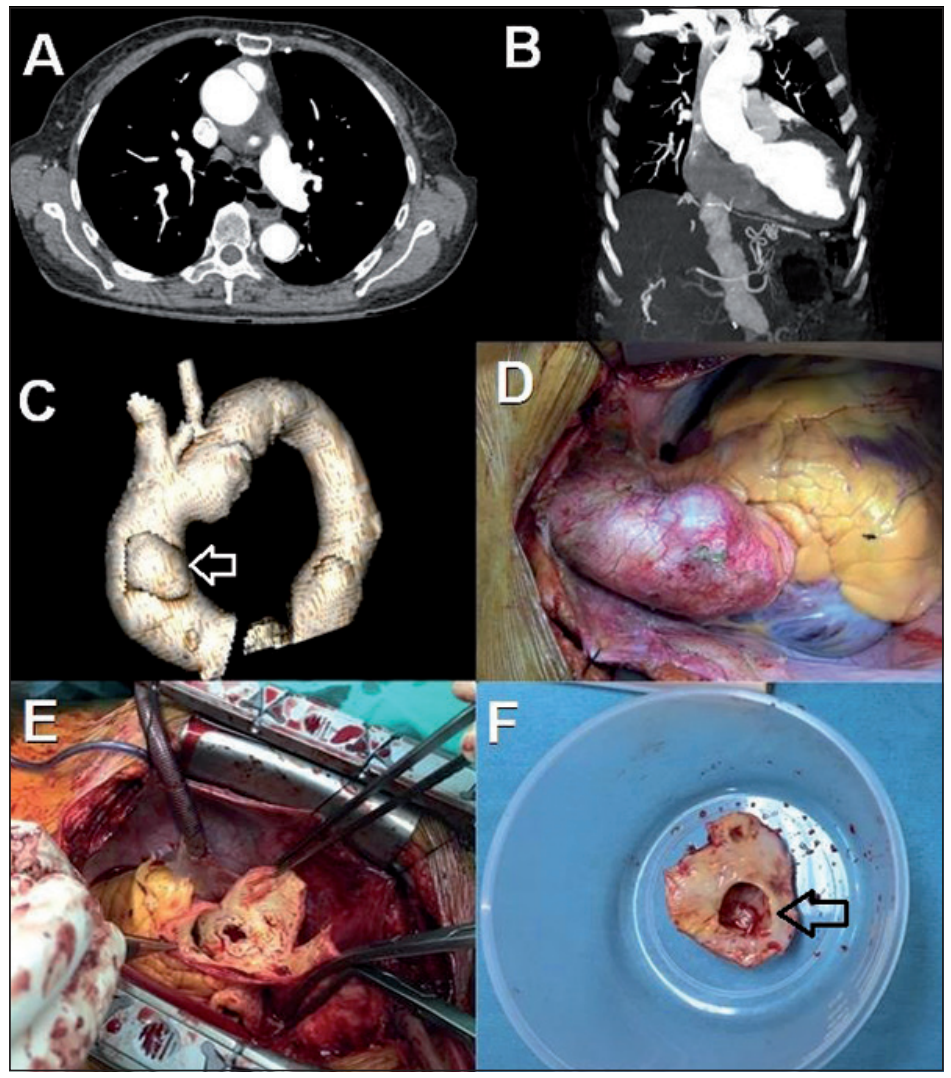

Figuras 2. A-B: Angio-TC que evidencia úlcera penetrante de aorta ascendente. C: Reconstrucción 3-D de úlcera penetrante de aorta ascendente (flecha). D-E-F: Imágenes intraoperatoria que evidencian úlcera penetrante de aorta ascendente (flecha). 
nariografía que no mostró lesiones significativas y ecocardiografía que demostró hipertrofia ventricular izquierda, disfunción diastólica tipo II y dilatación de aurícula izquierda.

Debido al alto riesgo que representaba la UPAA, se decidió cirugía, que se realizó 5 semanas posterior a la cirugía abdominal. Se abordó por esternotomía y se realizó bajo CEC reemplazo con prótesis de dacron Gelware ${ }^{\mathrm{TM}} \# 26$. Se observó en el intraoperatorio una lesión pulsátil de alrededor de dos centímetros de diámetro en la cara anterior de la AA y, al diseccionar la AA, se encontraron tres úlceras, una de ellas a un centímetro del ostium coronario derecho y no se observó disección aórtica. (Figura 2 D, E y F).

La paciente evolucionó favorablemente y se dio de alta a los siete días postoperada difiriéndose la resolución del aneurisma de la aorta abdominal.

El estudio anatomopatológico confirmó UPAA. Actualmente, a un año y diez meses de seguimiento, la paciente continúa sus controles en Cardiología sin presentar sintomatología cardiovascular.

\section{Discusión}

La UPAA es una patología que tiene una leve predilección por el sexo masculino (60\% de los casos $)^{3}$, generalmente se asocia a HTA y enfermedad aterosclerótica avanzada que dan a lugar a la disrupción de la capa intima de la aorta ${ }^{6,8-10}$. Se localiza preferentemente en la aorta torácica descendente $(85-95 \%)$, siendo inusual su localización en la AA $(1,8 \%)$.

Las manifestaciones clínicas de los pacientes con UPAA dependen de la evolución y complicaciones que ésta presente. Así, una UPAA que evolucione hacia una DAo, un HI o rotura, debutará como SAA, mientras que un paciente que únicamente presente UPAA no siempre debutará con síntomas cardiovasculares. En nuestro trabajo presentamos ambas modalidades de presentación, primero una paciente sintomática con SAA y otra asintomática cardiovascular con diagnóstico imagenológico de UPAA con alto riesgo de rotura.

El principal síntoma del SAA es el dolor torácico que se localiza en la región anterior del tórax, cuello y mandíbula; puede irradiarse a la región interescapular y se caracteriza por ser intenso, inicio súbito, punzante o desgarrante ${ }^{11}$. También se puede acompañar de rotura aórtica, insuficiencia cardíaca o síndromes de mala perfusión. En el SAA, una clasificación utilizada es la de Stanford ${ }^{12}$, diferenciando aquellas DAo que afectan la AA (Stanford A) de aquellas que comprometen el resto de la aorta (Stanford B).

Para el diagnóstico es clave mantener un alto índice de sospecha para solicitar los exámenes imageneológicos que permitan diagnosticar a tiempo y, así, poder realizar un diagnóstico diferencial con patologías que cursan con dolor torácico agudo como IAM, tromboembolismo pulmonar o patología gastroesofágica. Los exámenes más utilizados son el Angio-TC y la ecocardiografía transesofágica, que evidencian una imagen sacular externa y con márgenes irregulares de calcificación de la íntima. En ambos casos presentados, el Angio-TC fue el método diagnóstico, más aun en el segundo caso, en donde la evolución fue totalmente asintomática desde el punto de vista cardiovascular.

El tratamiento del SAA dependerá de la localización de las lesiones, de este modo, está bien determinado que las lesiones (UPA, DAo o HI) ubicadas en la AA tienen indicación quirúrgica con reemplazo protésico del segmento afectado por el alto riesgo de rotura aórtica ${ }^{13-17}$; incluso en pacientes asintomáticos. Mientras que las lesiones de la aorta descendente pueden tratarse por vía endovascular o quirúrgicamente, dependiendo del tipo de lesión y la estabilidad hemodinámica del paciente.

De acuerdo al "International Registry of acute aortic dissections" (IRAD), el pronóstico de los pacientes intervenidos quirúrgicamente de SAA, con lesiones Stanford A, ha mejorado significativamente en el tiempo, en cuanto a mortalidad a corto plazo, desde $25 \%$ a $18 \%$, siendo el principal predictor las comorbilidades del paciente y las complicaciones que estos presenten ${ }^{15}$. Las principales morbilidades son: hemorragia, IAM, accidente cerebrovascular, sepsis, arritmias, entre otras $^{18}$. La supervivencia de pacientes intervenidos quirúrgicamente por SAA a 1 y 3 años de seguimiento es de $96 \%$ y $91 \%$, respectivamente, siendo notablemente superior al tratamiento médico, aun en pacientes añosos y de alto riesgo quirúrgico ${ }^{15-17,19}$.

Las guías de la Europan Society of Cardiology $y$ la American College of Cardiology Foundation/ American Heart Association recomiendan la cirugía como tratamiento en estas lesiones cuando afecten a la aorta ascendente ${ }^{18,20}$. 
En conclusión, la UPA es una entidad clínica infrecuente, observable en 5\% de los SAA y sólo $1,8 \%$ de las veces se localiza en la AA. Antecedentes de HTA y una avanzada ateroesclerosis son críticas en la pérdida de la integridad de la pared aórtica. Es clave tener una alta sospecha para su diagnóstico, dado que casi la mitad de las veces la UPA se presenta de forma asintomática y su tratamiento es la cirugía, dado el alto riesgo de progresar hasta la rotura.

\section{Referencias}

1. Lansman S, Saunders P, Malekan R, Spielvogel D. Acute aortic syndrome. J Thorac Cardiovasc Surg 2010; 140 (6): S92-7.

2. Bayona A, Vallejo E, Hernández-Estefanía R, Bastarrika G, Rábago G. Penetrating ascending aortic ulcer in an asymptomatic patient. An Sist Sanit Navar 2012; 35 (2): 323-7.

3. Vega J, Zamorano J, Pereira N, Galleguillos A. Síndrome aórtico agudo: Revisión de la literatura y actualización del tema. Rev Med Chile 2014; 142 (3): 344-52.

4. Troxler M, Mavor A, Homer-Vanniasinkam S. Penetrating atherosclerotic ulcers of the aorta. Br J Surg 2001; 88 (9): 1169-77.

5. Nathan D, Boonn W, Lai E, Wang G, Desai N, Woo E, et al. Presentation, complications, and natural history of penetrating atherosclerotic ulcer disease. J Vasc Surg 2012; 55 (1): 10-5.

6. Wada H, Sakata N, Tashiro T. Clinicopathological study on penetrating atherosclerotic ulcers and aortic dissection: distinct pattern of development of initial event. Heart Vessels 2016; 31 (11): 1855-61.

7. Bradley T, Bowdin S, Morel C, Pyeritz R. The Expanding Clinical Spectrum of Extracardiovascular and Cardiovascular Manifestations of Heritable Thoracic Aortic Aneurysm and Dissection. Can J Cardiol 2016; 32 (1): 86-99.

8. Bonaca M, O'Gara P. Diagnosis and Management of Acute Aortic Syndromes: Dissection, Intramural Hematoma, and Penetrating Aortic Ulcer. Curr Cardiol Rep 2014; 16 (10): 536.
9. Patatas K, Shrivastava V, Ettles D. Penetrating atherosclerotic ulcer of the aorta: A continuing debate. Clinical Radiol 2013; 68 (8): 753-9.

10. Singhal P, Lin Z. Penetrating Atheromatous Ulcer of Ascending Aorta: A Case Report and Review of Literature. Heart Lung Circ 2008; 17 (5): 380-2.

11. Hagan P, Nienaber C, Isselbacher E, Bruckman D, Karavite $\mathrm{D}$, Russman $\mathrm{P}$, et al. The International Registry of Acute Aortic Dissection (IRAD). New insights in to an old disease. JAMA 2000; 283 (7): 897-903.

12. Daily P, Trueblood H, Stinson E, Wuerflein R, Shumway N. Management of acute aortic dissections. Ann Thorac Surg 1970; 10 (3): 237-47.

13. Singhal $\mathrm{P}$, Lin Z. Penetrating atheromatous ulcer of ascending aorta: A case report and review of literature. Heart Lung Circ 2008; 17 (5): 380-2.

14. Corvera J. Acute aortic syndrome. Ann Cardiothorac Surg 2016; 5 (3): 188-93.

15. Berretta P, Patel H, Gleason T, Sundt T, Myrmel T, Desai N, et al. IRAD experience on surgical type A acute dissection patients: results and predictors of mortality. Ann Cardiothorac Surg 2016; 5 (4): 346-51.

16. Mussa F, Horton J, Moridzadeh R, Nicholson J, Trimarchi S, Eagle K. Acute Aortic Dissection and Intramural Hematoma: A Systematic Review. JAMA 2016; 316 (7): 754-63.

17. Myrmel T, Larsen M, Bartnes K. The International Registry of Acute Aortic Dissections (IRAD) - experiences from the first 20 years. Scand Cardiovasc J 2016; 50 (56): 329-33.

18. Hiratzka L, Bakris L, Beckman J, Bersin R, Carr V, Casey D. 2010 ACCF/AHA/AATS/ACR/ASA/SCA/SCAI/SIR/ STS/SVM guidelines for the diagnosis and management of patients with Thoracic Aortic Disease. Circulation. 2010; 121 (13): e266-369.

19. Castaño M, Gualis J, Martínez-Comendador J, Martín E, Maiorano P, Castillo L. Emergent aortic surgery in octogenarians: is the advanced age a contraindication?. J Thorac Dis 2017; 9 (6): S498-S507.

20. Erbel R, Aboyans V, Boileau C, Bossone E, Di Bartolomeo R, Eggebrecht H, et al. 2014 ESC Guidelines on the diagnosis and treatment of aortic diseases. Eur Heart J 2014; 35 (41): 2873-926. 